\title{
Differing efficacies of autologous platelet-rich plasma treatment in reducing pain following rotator-cuff injury in a single patient
}

This article was published in the following Dove Press journal: Journal of Pain Research

\section{Damien P Kuffler}

Institute of Neurobiology, Medical Sciences Campus, University of Puerto Rico, San Juan 0090I, Puerto Rico
Correspondence: Damien P Kuffler Institute of Neurobiology, 20I Boulevard del Valle, San Juan 00909I, Puerto Rico Tel +I 787 72I 0778 Email dluffler@gmail.com

\begin{abstract}
Rotator-cuff tears (RCTs) are typically associated with chronic pain. The most common treatment for reducing pain is the injection of cortisone into the injury site. An alternative and increasingly used technique is the injection of autologous platelet-rich plasma (PRP). A limitation of cortisone is its pain relief is short-lived, while PRP has the limitation of providing pain relief to only about $50 \%$ of patients, making its efficacy questioned, although when it is effective, its efficacy is longer than cortisone. Little is known about what accounts for these limitations. This paper presents results from a patient with RCTs causing excruciating pain who received an injection of PRP into that shoulder resulting in complete pain elimination that was ongoing at 2 years and 2 months. When 7-month severe posttrauma pain associated with the RCT developed in the contralateral shoulder, PRP prepared the same way and injected by the same person who performed the first injection provided no pain relief. However, a subsequent single cortisone injection resulted in complete pain elimination, which was ongoing at $>15$ months. These observations indicate that PRP can have inconsistent effects in reducing pain, not only between patients but also within the same patient. Further, although the pain relief induced by cortisone is normally not long-lasting, when given following a PRP injection, it can induce complete pain relief lasting $>15$ months. This paper discusses possible reasons for the variability in PRP efficacy on pain relief and addresses the possibility that when administered together, PRP and cortisone may act in a complementary manner, leading to significantly greater and longer-lasting pain relief.
\end{abstract}

Keywords: PRP, corticosteroids, RCTs, pain, pain reduction, platelets, inflammation, nerve injury

\section{Introduction}

Rotator-cuff tears (RCTs) caused by trauma are a common occurrence affecting millions of people worldwide. RCTs cause severe pain, which is exacerbated by arm movement and significantly reduces an individual's quality of life. In addition, the weakness caused by RCTs results in a major reduction in range of arm movement and arm strength, which also has a significant negative effect on an individual's quality of life, such as by making it difficult to dress oneself and to sleep. Therefore, it is essential to develop techniques that promote enhanced tendon repair and more reliable, significant, and long-lasting pain relief.

\section{Causes of RCT pain}

One cause of pain associated with RCTs is the development of inflammation, triggered in part by injury-induced platelet release of proinflammatory cytokines, such as IL1 $\beta$, 
IL6, and TNF $\alpha{ }^{1,2}$ These cytokines also evoke persistent pathologic pain by directly activating nociceptive sensory neurons and causing nerve-injury-induced central sensitization. ${ }^{3,4}$ In addition, proinflammatory cytokines induce the upregulation of gene expression in nociceptive neuron axons and soma of voltage-gated sodium 1.8 ion channels, causing them to become chronically ectopically electrically active and thus sending continuous pain signals to the brain., Finally, inflammation and pain are triggered by lysed red blood cells at an injury site, releasing oxygen free radicals, ${ }^{7}$ with white blood cells releasing both oxygen free radicals and matrix metalloproteases. ${ }^{8}$

These pain etiologies suggest that one approach to eliminating chronic pain associated with RCTs is to eliminate chronic inflammation. A technique for eliminating inflammation is to induce enhanced inflammation as a means of triggering the reinitiation of the normal wound-healing process, which involves an increasing level of inflammation that then decreases until it is completely eliminated. This can be accomplished by the application of PRP, which releases both pro- and anti-inflammatory cytokines. Alternatively, inflammation can be eliminated by the application of antiinflammatory cytokines, such as IL1-receptor antagonist, IL10, IL11, and IL13. ${ }^{2}$ IL10 also reduces inflammation by blocking the synthesis and release of proinflammatory factors, upregulating the synthesis and release of additional antiinflammatory cytokines and simultaneously downregulating the expression of genes for proinflammatory cytokine receptors. ${ }^{4}$ The efficacy of the administration of IL10 is shown by its suppressing the development of spinally mediated pain facilitation in various animal pain models, such as peripheral neuritis, spinal cord excitotoxic injury, and peripheral nerve injury. ${ }^{9}$ The end result of each approach is to induce the transition of a wound from a pro- to an anti-inflammatory state that does not evoke pain.

Another mechanism for eliminating inflammation-associated pain is the application of IL10, which is released by platelets and induces the downregulation of injury-induced gene expression for the voltage-gated sodium 1.8 ion channel in primary nociceptive neuron axons and somas. ${ }^{5,6,10}$ The loss of this ion channel results in the cessation of nociceptive neuron chronic ectopic electrical activity and thereby the loss of chronically evoked pain.

\section{Techniques for promoting pain reduction following RCTs \\ Corticosteroid injections}

Due to their anti-inflammatory actions, corticosteroid injections are presently the method of choice to promote relief of pain associated with RCTs. However, there is the question of the reliability of corticosteroid injections to provide relief from pain due to RCTs, which have a success rate of $60 \%-100 \%{ }^{11-13}$ Another limitation of cortisone use is that the level of pain reduction varies from $50 \%$ to $84 \% .^{14,15}$ Part of this variation may be due to lack of precision of the injurysite injection, with higher pain-suppression efficacy being obtained when using image-guidance tools for the injection, such as magnetic resonance imaging, ${ }^{16}$ dynamic fluoroscopic control, ${ }^{17}$ and ultrasound. ${ }^{18}$ Another limitation is that although cortisone's analgesic influence generally lasts 4-12 weeks ${ }^{19-22}$ and may be effective for up to 6 months, its pain relief is generally not long-term. ${ }^{13,23,24}$ It is important to note that a repeat corticosteroid injection after 2 or 3 weeks does not increase the duration of the analgesic effects seen after 6 months. ${ }^{11}$

\section{PRP injections}

Numerous studies have tested the efficacy of injecting autologous platelet-rich plasma (PRP) into the site of RCTs to induce tendon healing and reduce chronic pain..$^{25}$ The use of PRP for pain relief has two major limitations. First, a number of studies and analyses of the published literature have indicated a lack of reliable evidence that PRP provides effective pain relief. ${ }^{26-33}$ In general, this conclusion is ascribed to the study samples being small, there being few controlled trials, and many results being anecdotal. However, a recent thorough meta-analysis of randomized controlled trials and a well-controlled study concluded that PRP improves rotatorcuff healing rates and functional outcomes and reduces pain levels. ${ }^{34,35}$ Several recent studies have also found that combining PRP with surgical tendon repairs significantly improves tendon healing while reducing retear rates, which should also contribute to pain reduction. ${ }^{36-38} \mathrm{~A}$ second limitation is that when pain relief was observed, it was not long-lasting, generally lasting only 6 months, ${ }^{21,29,39-41}$ although complete pain relief $>12$ months has been reported. ${ }^{42,43}$

Although results indicating that PRP provides pain relief are questioned, the failure of PRP to provide pain relief may result not from its inability to be effective, but from differences in the composition of the PRP applied in different studies. Such differences can arise due to the different devices used to separate PRP from whole blood. Different PRP-separation devices result in platelet concentrations that may be less than that of whole blood to sixfold as high, variations in $\mathrm{pH},{ }^{44}$ and differences in leukocyte and growth-factor concentrations that can vary by fivefold. ${ }^{44-47}$

Another significant factor influencing PRP composition is the physiological status of the person from whom blood is 
collected. As their physiological status changes, so does their blood composition, such as having an elevated leukocyte concentration. Elevated whole-blood leukocyte concentration can result in PRP with an elevated concentration of leukocytes. This is important, because leukocyte-rich PRP is detrimental to the proliferation of some stem/progenitor cells, leading to impaired tissue healing, because leukocytes inhibit the release of growth factors, such as VEGF, EGF, TGF $\beta_{1}$, and PDGF, increasing catabolic cytokine concentrations and causing inflammation and apoptosis. ${ }^{47-50}$ Therefore, differences in PRP composition due to different PRP-separation devices or physiological changes in the patient may account for some of the variability in the efficacy of PRP on pain reduction. ${ }^{51,52}$

Other factors may also alter the efficacy of PRP in inducing pain relief. While the optimal time to apply PRP in rats is immediately postinjury, ${ }^{53}$ it is not known if this is optimal for the clinical application of PRP. Although multiple PRP injections appear to promote pain reduction, ${ }^{41,45}$ there are no comparative data about the relative efficacy of one vs more PRP injections. All combined, these many variables can result in PRP having concentrations of factors that vary by more than 30 -fold. Until it is known which factors and at what concentrations are critical for reducing inflammation and pain, each difference in PRP composition must be considered when evaluating whether PRP can or cannot reduce/ eliminate inflammation and pain. Finally, little is known about whether the efficacy of PRP can be increased if it is injected in combination with, prior to, or after, an injection of an anti-inflammatory mediator, such as a corticosteroid.

\section{Comparative influences of PRP and corticosteroids}

The relative influences of PRP vs corticosteroid injections on pain relief vary in both the extent and duration of pain relief. While corticosteroid injections generally provide pain relief and the influence of PRP is more variable, ${ }^{32}$ corticosteroids may be more effective than autologous blood products in providing short-term (up to about 12 weeks) pain relief. ${ }^{54}$ For the intermediate term (6 months), autologous blood products appear to provide better therapeutic effect for pain relief than corticosteroids. ${ }^{54}$ However, for longer times, such as 6 months to 1 year, while there are reported to be no differences in the extent of reduction in pain levels, ${ }^{55,56} \mathrm{PRP}$ has been reported to provide significantly greater pain relief. ${ }^{52,57}$ For pain relief due to osteoarthritis, corticosteroids are more effective than PRP, with higher corticosteroid doses inducing longer-lasting pain relief. ${ }^{58}$ However, PRP treatment has also been found to induce significantly more pain reduction for patients than is induced by corticosteroid injections, ${ }^{59}$ with the duration of
PRP pain relief being significantly longer lasting than that of corticosteroids. ${ }^{56,60-62}$ Therefore, in general, PRP induces a greater degree of longer-lasting pain relief than corticosteroids. However, the small samples and limited number of high-quality randomized controlled trials mean that further studies are required to validate these conclusions.

\section{Pain development in injured shoulder followed by subsequent development of RCT and pain in contralateral shoulder}

Patients with a partial- or full-thickness RCT ipsilaterally to the trauma have a $36 \%$ risk of developing a full-thickness tear of the contralateral rotator cuff, with noticeable deficits in shoulder function related to daily living activities, even if the tear is asymptomatic. ${ }^{63-65}$ The size of an RCT tear is an important factor in the development of increased symptoms. In contrast, patients with a partial-thickness tear or a normal rotator cuff on the painful side have rates of full-thickness tears that are low on the asymptomatic side of $4.3 \%$ and $0.5 \%$, respectively.

\section{Case report}

A 67-year-old male had a motor scooter accident causing him to fall to the ground, landing on his shoulder. Due to excruciating pain, the subject consumed ibuprofen (200 $\mathrm{mg}$ ) and hydrocodone $7.5(\mathrm{mg})$ up to six times per day as required. Although the subject had surgery to repair the damage scheduled, he wanted to try an alternative to surgery. As such, about 2.5 months following the accident, the subject received one injection of PRP into the right shoulder in the hope that the PRP would alleviate the pain and induce healing. By 7 days following the PRP injection, the subject's pain had decreased significantly, and by the end of the following 7 days he had stopped use of all analgesics. The scheduled surgery was canceled. After an additional 3 months, the patient had slight but annoying residual pain. The patient received a corticosteroid injection, and after 3 weeks was about $98 \%$ pain-free. The patient remained virtually pain-free more than 2 years and 2 months following the PRP injection.

About 7 months following the accident, the subject began to feel pain in the left shoulder contralaterally to the one that had caused the extreme pain. Although the pain was intense, it was not as severe as the pain of the first shoulder. Magnetic resonance imaging indicated that the pain was due to a partial tear of the left supraspinatus muscle. The pain was treated with ibuprofen $400 \mathrm{mg}$ four times per day and hydrocodone $7.4 \mathrm{mg}$ every day as needed, but the pain did not decrease with time. 
After about 3 months of persistent pain, the patient received an injection of PRP into the second shoulder. By 1.5 months following the PRP injection, no pain relief had developed and the pain persisted at a relatively constant level for about another 5 weeks. Seeking pain relief, the subject received a corticosteroid injection into the shoulder, and within 12 days the pain had disappeared and the patient went off all analgesics. At 15 months following the corticosteroid injection, the second shoulder remained almost $100 \%$ pain-free. The subject still suffers some muscle weakness and there are some tasks, such as lifting heavy objects above his head, which he is unable to do. However, the patient considers the treatment a complete success. The patient provided written informed consent to have the case details published.

\section{Discussion}

Many studies have reported success using PRP injections to reduce or eliminate chronic pain associated with RCTs and other tendon injuries. ${ }^{21,39-41}$ However, systematic studies comparing the results of studies reporting the efficacy of PRP injection in providing pain relief have generally not found any statistical evidence supporting the claim that PRP is effective. ${ }^{26-28,31-33}$ These studies concluded that the major faults with studies claiming PRP efficacy in providing pain relief were their small samples and being uncontrolled. However, these comparative studies typically did not take into account factors that could significantly influence PRP efficacy. ${ }^{44,45,47,51,52}$ As such, the results from comparative studies should be considered valid only if they come from individuals on whom the same technique and device was used to prepare and administer the PRP, ${ }^{16-18,66-68}$ PRP was administered at the same time posttrauma, similar volumes of PRP were administered, PRP was applied to the same type of tissue, whether the physiological status of the patients was taken into account, and whether the study was clinical or on an animal model. Although these are rigorous requirements, only by comparing standardized studies will it be possible to determine whether PRP is truly effective. Comparing results from different standardized studies involving different sets of parameters should provide insights into which variables influence the potential efficacy of PRP on pain relief.

This paper discusses the results of a patient who developed excruciating chronic pain associated with RCTs in one shoulder and for whom the pain was virtually eliminated by an injection of PRP. The shoulder remained virtually $100 \%$ pain-free after 2 years and 2 months. At 7 months after the initial injury, severe chronic pain developed in the contralateral shoulder. An injection of PRP, prepared using the same technique as the first batch of PRP and administered by the same person, provided no pain relief. However, a subsequent injection of corticosteroid into that shoulder led to the elimination of the pain. That shoulder remained virtually $100 \%$ pain-free 17 months after the PRP injection and 15 months following the corticosteroid injection.

These results show that injections of PRP into the same patient, even when prepared using the same technique and administered by the same person, can result in complete or no pain elimination. This finding eliminates the potential that the differences in PRP influences were due to differences in how the PRP was prepared and administered. They suggest that the different outcomes resulted from injecting PRP with different PRP compositions are due to a change in the patient's physiological status, platelet count, and/or platelet factor content. These observations indicate that before reliable influences of PRP on pain relief can be anticipated, it is essential to determine the PRP composition required to eliminate chronic pain and the mechanisms by which pain is reduced/eliminated.

While tissue injury is associated with the development of inflammation and pain, the normal healing process leads to the reduction and eventual elimination of inflammation and its associated pain. However, for unknown reasons, injury sites can become stuck in a state of chronic inflammation, resulting in the generation of chronic pain. As mentioned, a number of mechanisms may eliminate chronic inflammation and its associated chronic pain, most of which involve the application of proinflammatory cytokines to trigger reinitiation of the wound-healing process, causing inflammation to increase followed by its elimination, anti-inflammatory cytokines to force the transition of a wound site directly from a pro- to an anti-inflammatory state, and an anti-inflammatory cytokine to eliminate spontaneous nociceptive neuron ectopic electrical activity.

When considering the potential efficacy of PRP and cytokines in providing pain relief, an important question is why when PRP appears to have the capacity to reduce pain, it does so inconsistently, and why when it is effective, its influence may be incomplete and not permanent. As suggested, the answer may lie in the variability of PRP composition such that it does not always induce the same degree or duration of inflammation suppression, and thus not the same degree of pain suppression. Variability in 
PRP composition may arise from different devices used to separate PRP from whole blood, yielding PRP with considerably different platelet concentrations, ratios of activated vs nonactivated platelets, $\mathrm{pH}$, and concentration of platelet-contained factors. Each of these variables can greatly affect the capability of PRP to reduce inflammation and pain. Therefore, the composition of PRP-released factors may not be sufficient to trigger reinitiation of the pro- and anti-inflammatory process or entirely eliminate the proinflammatory state, which is required to eliminate inflammation permanently and thus pain.

Further complicating the reliability of the ability of PRP to reduce inflammation and pain is that the composition of PRP can vary greatly depending on inherent factors of the individual from whom PRP is prepared. These variables include the person's normal platelet count, heath status, such as illness or disease, levels of mental and physical stress, hormone levels, and drug and alcohol consumption. As such, the composition of PRP prepared from whole blood from the same individual using the same device can vary considerably over time. For the patient discussed in this paper, the composition of his PRP might have changed sufficiently between the first and second preparation of PRP, thus allowing it to eliminate the pain in his first shoulder completely, but failing to reduce pain when it was applied to the second shoulder.

Given these variables, the question remains about what might be done to eliminate chronic pain when PRP does not reduce or completely eliminate it. Is there an alternative approach that might be combined with the application of PRP to induce complete and permanent pain relief? Corticosteroid injections are generally considered effective in providing pain relief, ${ }^{11,12,14,15,69}$ although doubts remain as to their efficacy in reducing pain due to RCTs. ${ }^{13}$ Corticosteroids act to reduce inflammation and pain via multiple mechanisms. First, they markedly reduce production of the proinflammatory cytokines IFN $\gamma, \mathrm{TNF} \alpha$, IL1, and IL12. ${ }^{1}$ Second, similarly to platelets, corticosteroids suppress inflammation by increasing the synthesis and release of IL $10,{ }^{70}$ which leads to the upregulation of synthesis and release of additional anti-inflammatory cytokines. ${ }^{4}$ However, these actions do not always eliminate the proinflammatory environment entirely. Therefore, over time the residual inflammation may trigger renewed synthesis and release of proinflammatory cytokines, leading to a further increase in inflammation and increased pain. As such, a significant limitation of corticosteroids in providing pain relief appears to be their failure to suppress inflammation completely, resulting in the relatively short duration of their pain relief, generally not more than 6 months, ${ }^{11,13,23,24}$ and the reoccurrence of pain.

The present data raises the question of, why when PRP was injected into the second shoulder, it did not eliminate the pain, but a subsequent corticoid injection led to complete and long-lasting pain relief. Several possibilities could have acted singly or in combination. One is that the corticosteroid injection suppressed the synthesis and release of proinflammatory cytokines that had not been blocked or suppressed by the PRP injection. Corticosteroid promotion of the release of the anti-inflammatory cytokine IL10 could have reduced the proinflammatory state of the RCTs. Finally, corticosteroidinduced released IL10 may have suppressed the remaining spontaneous ectopic electrical activity of nociceptive neurons that was causing the chronic pain. Therefore, the combined action of PRP and the corticosteroid could have completely and permanently eliminated all inflammation, as well as spontaneous nociceptive ectopic electrical activity, leading to complete and permanent pain suppression. Further studies are required to determine whether when PRP fails or only partially reduces pain, reliable, complete, and long-lasting pain reduction can be induced by injection of a corticosteroid.

\section{Conclusion}

This study found that an injection of PRP into the shoulder of an individual with RCTs causing severe chronic pain virtually eliminated the pain, while an injection of PRP 7 months later into the other shoulder that was also causing extreme pain had no impact on the pain level. However, for the second shoulder, a subsequent injection of corticosteroid led to the virtual elimination of the pain. The pain elimination for the two shoulders lasted more than 2 years and 2 months and 15 months respectively. These results indicate that even when the same device is used to prepare PRP and the PRP administered by the same individual using the same method, varying levels of pain reduction can result. This suggests that variations in a patient's physiological status may sufficiently alter their whole blood, leading to such a degree of change in the composition of their PRP that it is not effective in suppressing inflammation and pain. Because the pain relief provided by corticosteroids is normally short-lived, the complete and long-term pain relief achieved by the second shoulder when corticosteroid was administered following PRP suggests that this resulted 
from the combined actions of PRP and corticosteroids inducing a permanent transition of the RCT site from pro- to anti-inflammatory, which would lead to complete and permanent pain relief. Further studies are required to determine whether consistent, complete, and long-term pain relief is provided by combining an injection of PRP with corticosteroids, or by merely increasing the concentration of platelet-released factors.

\section{Disclosure}

The author reports no conflicts of interest in this work.

\section{References}

1. Petrovsky N, McNair P, Harrison LC. Diurnal rhythms of proinflammatory cytokines: regulation by plasma cortisol and therapeutic implications. Cytokine. 1998;10(4):307-312.

2. Xie WR, Deng H, Li H, et al. Robust increase of cutaneous sensitivity, cytokine production and sympathetic sprouting in rats with localized inflammatory irritation of the spinal ganglia. Neuroscience. 2006;142(3):809-822.

3. Watkins LR, Milligan ED, Maier SF. Glial proinflammatory cytokines mediate exaggerated pain states: implications for clinical pain. Adv Exp Med Biol. 2003;521:1-21.

4. Zhang JM, An J. Cytokines, inflammation, and pain. Int Anesthesiol Clin. 2007;45(2):27-37.

5. Bedi SS, Yang Q, Crook RJ, et al. Chronic spontaneous activity generated in the somata of primary nociceptors is associated with painrelated behavior after spinal cord injury. J Neurosci. 2010;30(44): 14870-14882.

6. Yang Q, Wu Z, Hadden JK, et al. Persistent pain after spinal cord injury is maintained by primary afferent activity. $J$ Neurosci. 2014;34(32): 10765-10769.

7. Jiang N, Tan NS, Ho B, Ding JL. Respiratory protein-generated reactive oxygen species as an antimicrobial strategy. Nat Immunol. 2007;8(10):1114-1122.

8. Scott A, Khan KM, Roberts CR, Cook JL, Duronio V. What do we mean by the term "inflammation"? A contemporary basic science update for sports medicine. Br J Sports Med. 2004;38(3):372-380.

9. Wieseler-Frank J, Maier SF, Watkins LR. Glial activation and pathological pain. Neurochem Int. 2004;45(2-3):389-395.

10. Shen KF, Zhu HQ, Wei XH, et al. Interleukin-10 down-regulates voltage gated sodium channels in rat dorsal root ganglion neurons. Exp Neurol. 2013;247:466-475.

11. Gialanella B, Prometti P. Effects of corticosteroids injection in rotator cuff tears. Pain Med. 2011;12(10):1559-1565.

12. Speed CA. Fortnightly review: corticosteroid injections in tendon lesions. BMJ. 2001;323(7309):382-386.

13. Buchbinder R, Green S. Corticosteroid injections for shoulder pain. Cochrane Database Syst. Rev. 2003;(1):CD004016.

14. Blair B, Rokito AS, Cuomo F, Jarolem K, Zuckerman JD. Efficacy of injections of corticosteroids for subacromial impingement syndrome. J Bone Joint Surg Am. 1996;78(11):1685-1689.

15. Plafki C, Steffen R, Willburger RE, Wittenberg RH. Local anaesthetic injection with and without corticosteroids for subacromial impingement syndrome. Int Orthop. 2000;24(1):40-42.

16. Groom LM, White NA, Adams MN, Barrett JG. Accuracy of open magnetic resonance imaging for guiding injection of the equine deep digital flexor tendon within the hoof. Vet Radiol Ultrasound. 2017;58(6):671-678.

17. Rastogi AK, Davis KW, Ross A, Rosas HG. Fundamentals of Joint Injection. AJR Am J Roentgenol. 2016;207(3):484- 494.
18. Berkoff DJ, Miller LE, Block JE. Clinical utility of ultrasound guidance for intra-articular knee injections: a review. Clin Interv Aging. 2012;7:89-95.

19. Ang TW. The effectiveness of corticosteroid injection in the treatment of plantar fasciitis. Singapore Med J. 2015;56(8): $423-432$.

20. Crawford F, Atkins D, Young P, Edwards J. Steroid injection for heel pain: evidence of short-term effectiveness. A randomized controlled trial. Rheumatology. 1999;38(10):974-977.

21. Rha DW, Park GY, Kim YK, Kim MT, Lee SC. Comparison of the therapeutic effects of ultrasound-guided platelet-rich plasma injection and dry needling in rotator cuff disease: a randomized controlled trial. Clin Rehabil. 2013;27(2):113-122.

22. Thanasas C, Papadimitriou G, Charalambidis C, Paraskevopoulos I, Papanikolaou A. Platelet-rich plasma versus autologous whole blood for the treatment of chronic lateral elbow epicondylitis: a randomized controlled clinical trial. Am J Sports Med. 2011;39(10): 2130-2134.

23. Faúndez J, Cotoras P, Irarrázaval S. Are intraarticular steroids effective for knee osteoarthritis? Medwave. 2016;16(Supp15):e6599.

24. Leadbetter WB. Anti-inflammatory therapy in sports injury. The role of nonsteroidal drugs and corticosteroid injection. Clin Sports Med. 1995;14(2):353-410.

25. Weber SC, Parise C. Regarding "Efficacy of platelet-rich plasma in arthroscopic repair of full-thickness rotator cuff tears: a meta-analysis". J Shoulder Elbow Surg. 2016;25(8):e246-e247.

26. de Vos RJ, Weir A, van Schie HT, et al. Platelet-rich plasma injection for chronic Achilles tendinopathy: a randomized controlled trial. JAMA. 2010;303(2):144-149.

27. Khan M, Bedi A. Cochrane in CORR (®): Platelet-rich Therapies for Musculoskeletal Soft Tissue Injuries (Review). Clin Orthop Relat Res. 2015;473(7):2207-2213.

28. Kim SJ, Lee SM, Kim JE, Kim SH, Jung Y. Effect of platelet-rich plasma with self-assembled peptide on the rotator cuff tear model in rat. J Tissue Eng Regen Med. 2017;11(1):77-85.

29. Kuffler DP. Coping with Phantom Limb Pain. Mol Neurobiol. 2018;55(1): 70-84.

30. Kuffler DP. Origins of Phantom Limb Pain. Mol Neurobiol. 2018;55(1):60-69.

31. Moraes VY, Lenza M, Tamaoki MJ, Faloppa F, Belloti JC. Platelet-rich therapies for musculoskeletal soft tissue injuries. Cochrane Database Syst Rev. 2014;(4):CD010071.

32. Ribeiro AG, Ricioli W, Silva AR, Polesello GC, Guimarães RP. PRP in the Treatment of Trochanteric Syndrome: A Pilot Study. Acta Ortop Bras. 2016;24(4):208-212.

33. Sampson S, Gerhardt M, Mandelbaum B. Platelet rich plasma injection grafts for musculoskeletal injuries: a review. Curr Rev Musculoskelet Med. 2008;1(3-4):165-174.

34. Flury M, Rickenbacher D, Schwyzer HK, et al. Does Pure Platelet-Rich Plasma Affect Postoperative Clinical Outcomes After Arthroscopic Rotator Cuff Repair? A Randomized Controlled Trial. Am J Sports Med. 2016;44(8):2136-2146.

35. Hurley ET, Lim Fat D, Moran CJ, Mullett H. The Efficacy of PlateletRich Plasma and Platelet-Rich Fibrin in Arthroscopic Rotator Cuff Repair: A Meta-analysis of Randomized Controlled Trials. Am J Sports Med. 2018;Epub 2018 Feb 21.

36. Barber FA, Hrnack SA, Snyder SJ, Hapa O. Rotator cuff repair healing influenced by platelet-rich plasma construct augmentation. Arthroscopy. 2011;27(8):1029-1035.

37. Cai YZ, Zhang C, Lin XJ. Efficacy of platelet-rich plasma in arthroscopic repair of full-thickness rotator cuff tears: a meta-analysis. J Shoulder Elbow Surg. 2015;24(12):1852-1859.

38. Meadows MC, Levy DM, Ferry CM, et al. Effects of Platelet-Rich Plasma and Indomethacin on Biomechanics of Rotator Cuff Repair. Am J Orthop. 2017;46(5):E336-E343. 
39. Kesikburun S, Tan AK, Yilmaz B, Yaşar E, Yazicioğlu K. Platelet-rich plasma injections in the treatment of chronic rotator cuff tendinopathy: a randomized controlled trial with 1-year follow-up. Am J Sports Med. 2013;41(11):2609-2616.

40. Patel S, Dhillon MS, Aggarwal S, Marwaha N, Jain A. Treatment with platelet-rich plasma is more effective than placebo for knee osteoarthritis: a prospective, double-blind, randomized trial. Am J Sports Med. 2013;41(2):356-364.

41. Sánchez M, Guadilla J, Fiz N, Andia I. Ultrasound-guided platelet-rich plasma injections for the treatment of osteoarthritis of the hip. Rheumatology. 2012;51(1):144-150.

42. Kon E, Buda R, Filardo G, et al. Platelet-rich plasma: intra-articular knee injections produced favorable results on degenerative cartilage lesions. Knee Surg Sports Traumatol Arthrosc. 2010;18(4):472-479.

43. Santiago-Figueroa J, Sosa IJ, Reyes O, Guzman H, Hernandez R, Kuffler DP. A novel technique for reducing and eliminating peripheral neuropathic pain: a clinical study. J. Pain Manag. 2011;4(4):387-394.

44. Fitzpatrick J, Bulsara MK, McCrory PR, Richardson MD, Zheng MH Analysis of Platelet-Rich Plasma Extraction: Variations in Platelet and Blood Components Between 4 Common Commercial Kits. Orthop J Sports Med. 2017;5(1):2325967116675272. doi:10.1177/2325967116675272.

45. Charousset C, Zaoui A, Bellaiche L, Bouyer B. Are multiple platelet-rich plasma injections useful for treatment of chronic patellar tendinopathy in athletes? a prospective study. Am J Sports Med. 2014;42(4):906-911.

46. Mazzucco L, Balbo V, Cattana E, Guaschino R, Borzini P. Not every PRP-gel is born equal. Evaluation of growth factor availability for tissues through four PRP-gel preparations: Fibrinet, RegenPRP-Kit, Plateltex and one manual procedure. Vox Sang. 2009;97(2):110-118.

47. Perut F, Filardo G, Mariani E, et al. Preparation method and growth factor content of platelet concentrate influence the osteogenic differentiation of bone marrow stromal cells. Cytotherapy. 2013;15(7):830-839.

48. Barber FA. Platelet-rich plasma for rotator cuff repair. Sports Med Arthrosc Rev. 2013;21(4):199-205.

49. Loibl M, Lang S, Brockhoff G, et al. The effect of leukocyte-reduced platelet-rich plasma on the proliferation of autologous adiposetissue derived mesenchymal stem cells. Clin Hemorheol Microcirc. 2016;61(4):599-614.

50. Zhang L, Chen S, Chang P, et al. Harmful Effects of Leukocyte-Rich Platelet-Rich Plasma on Rabbit Tendon Stem Cells In Vitro. Am J Sports Med. 2016;44(8):1941-1951.

51. Fitzpatrick J, Bulsara M, Zheng MH. The Effectiveness of Platelet-Rich Plasma in the Treatment of Tendinopathy: A Meta-analysis of Randomized Controlled Clinical Trials. Am J Sports Med. 2017;45(1):226-233.

52. Fitzpatrick J, Bulsara MK, O’Donnell J, McCrory PR, Zheng MH. The Effectiveness of Platelet-Rich Plasma Injections in Gluteal Tendinopathy: A Randomized, Double-Blind Controlled Trial Comparing a Single Platelet-Rich Plasma Injection With a Single Corticosteroid Injection. Am J Sports Med. 2018;46(4):933-939.

53. Çirci E, Akman YE, Şükür E, et al. Impact of platelet-rich plasma injection timing on healing of Achilles tendon injury in a rat model. Acta Orthop Traumatol Turc. 2016;50(3):366-372.

54. Qian X, Lin Q, Wei K, et al. Efficacy and Safety of Autologous Blood Products Compared With Corticosteroid Injections in the Treatment of Lateral Epicondylitis: A Meta-Analysis of Randomized Controlled Trials. PM R. 2016;8(8):780-791.
55. Mautner K, Blazuk J. Where do injectable stem cell treatments apply in treatment of muscle, tendon, and ligament injuries? PMR.2015;7(4 Suppl): S33-S40.

56. von Wehren L, Blanke F, Todorov A, et al. The effect of subacromial injections of autologous conditioned plasma versus cortisone for the treatment of symptomatic partial rotator cuff tears. Knee Surg Sports Traumatol Arthrosc. 2016;24(12):3787-3792.

57. Peerbooms JC, Sluimer J, Bruijn DJ, Gosens T. Positive effect of an autologous platelet concentrate in lateral epicondylitis in a double-blind randomized controlled trial: platelet-rich plasma versus corticosteroid injection with a 1-year follow-up. Am J Sports Med. 2010;38(2):255-262.

58. Chandrasekaran S, Lodhia P, Suarez-Ahedo C, et al. Symposium: evidence for the use of intra-articular cortisone or hyaluronic acid injection in the hip. J Hip Preserv Surg. 2016;3(1):5-15.

59. Gosens T, den Oudsten BL, Fievez E, van 't Spijker P, Fievez A. Pain and activity levels before and after platelet-rich plasma injection treatment of patellar tendinopathy: a prospective cohort study and the influence of previous treatments. Int Orthop. 2012;36(9):1941-1946.

60. Glanzmann MC, Audigé L. Platelet-rich plasma for chronic lateral epicondylitis: is one injection sufficient? Arch Orthop Trauma Surg. 2015;135(12):1637-1645.

61. Jain K, Murphy PN, Clough TM. Platelet rich plasma versus corticosteroid injection for plantar fasciitis: a comparative study. Foot. 2015;25(4):235-237.

62. Monto RR. Platelet-rich plasma efficacy versus corticosteroid injection treatment for chronic severe plantar fasciitis. Foot Ankle Int. 2014:35(4):313-318.

63. Liem D, Buschmann VE, Schmidt C, et al. The prevalence of rotator cuff tears: is the contralateral shoulder at risk? Am J Sports Med. 2014;42(4):826-830.

64. Merolla G, Paladini P, Saporito M, Porcellini G. Conservative management of rotator cuff tears: literature review and proposal for a prognostic. Prediction Score. Muscles Ligaments Tendons J. 2011;1(1): 12-19.

65. Yamaguchi K, Ditsios K, Middleton WD, et al. The demographic and morphological features of rotator cuff disease. A comparison of asymptomatic and symptomatic shoulders. J Bone Joint Surg Am. 2006;88(8):1699-1704.

66. Burke CJ, Adler RS. Ultrasound-Guided Percutaneous Tendon Treatments. AJR Am J Roentgenol. 2016;207(3):495-506.

67. Ferrero G, Fabbro E, Orlandi D, et al. Ultrasound-guided injection of platelet-rich plasma in chronic Achilles and patellar tendinopathy J Ultrasound. 2012;15(4):260-266.

68. Kuffler DP. Platelet-Rich Plasma Promotes Axon Regeneration, Wound Healing, and Pain Reduction: Fact or Fiction. Mol Neurobiol. 2015;52(2):990-1014.

69. Manchikanti L, Buenaventura RM, Manchikanti KN, et al. Effectiveness of therapeutic lumbar transforaminal epidural steroid injections in managing lumbar spinal pain. Pain Physician. 2012;15(3): E199-E245

70. Mozo L, Suárez A, Gutiérrez C. Glucocorticoids up-regulate constitutive interleukin-10 production by human monocytes. Clin Exp Allergy. 2004;34(3):406-412.
Journal of Pain Research

\section{Publish your work in this journal}

The Journal of Pain Research is an international, peer reviewed, open access, online journal that welcomes laboratory and clinical findings in the fields of pain research and the prevention and management of pain. Original research, reviews, symposium reports, hypothesis formation and commentaries are all considered for publication.
The manuscript management system is completely online and includes a very quick and fair peer-review system, which is all easy to use. Visit http://www.dovepress.com/testimonials.php to read real quotes from published authors. 